\title{
Erratum to: Determination of the integrated luminosity at HERA using elastic QED compton events
}

\section{The H1 Collaboration}

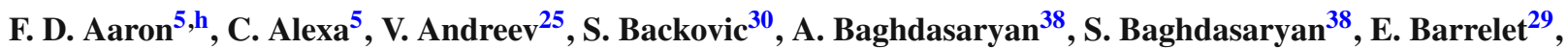
W. Bartel ${ }^{11}$, K. Begzsuren ${ }^{35}$, A. Belousov ${ }^{25}$, P. Belov ${ }^{11}$, J. C. Bizot ${ }^{27}$, V. Boudry ${ }^{28}$, I. Bozovic-Jelisavcic ${ }^{2}$, J. Bracinik ${ }^{3}$, G. Brandt ${ }^{11}$, M. Brinkmann ${ }^{11}$, V. Brisson ${ }^{27}$, D. Britzger ${ }^{11}$, D. Bruncko ${ }^{16}$, A. Bunyatyan ${ }^{13,38}$, A. Bylinkin ${ }^{24}$, L. Bystritskaya ${ }^{24}$, A. J. Campbell ${ }^{11}$, K. B. Cantun Avila ${ }^{22}$, F. Ceccopieri ${ }^{4}$, K. Cerny ${ }^{32}$, V. Cerny ${ }^{16,9}$, V. Chekelian ${ }^{26}$, J. G. Contreras ${ }^{22}$, J. A. Coughlan' ${ }^{6}$,J. Cvach ${ }^{31}$, J. B. Dainton ${ }^{18}$, K. Daum ${ }^{37, a, c}$, B. Delcourt ${ }^{27}$, J. Delvax ${ }^{4}$, E. A. De Wolf ${ }^{4}$, C. Diaconu ${ }^{21}$, M. Dobre ${ }^{12, j, k}$, V. Dodonov ${ }^{13}$, A. Dossanov ${ }^{12,26}$, A. Dubak ${ }^{30, f}$, G. Eckerlin ${ }^{11}$, S. Egli $^{36}$, A. Eliseev $^{25}$, E. Elsen ${ }^{11}$, L. Favart ${ }^{4}$, A. Fedotov ${ }^{24}$, R. Felst ${ }^{11}$, J. Feltesse ${ }^{10}$, J. Ferencei ${ }^{16}$, D.-J. Fischer ${ }^{11}$, M. Fleischer ${ }^{11}$, A. Fomenko ${ }^{25}$, E. Gabathuler ${ }^{18}$, J. Gayler ${ }^{11}$, S. Ghazaryan ${ }^{11}$, A. Glazov ${ }^{11}$, L. Goerlich ${ }^{7}$, N. Gogitidze ${ }^{25}$, M. Gouzevitch ${ }^{11, d}$, C. Grab ${ }^{40}$, A. Grebenyuk ${ }^{11}$, T. Greenshaw ${ }^{18}$, G. Grindhammer ${ }^{26}$, S. Habib ${ }^{11}$, D. Haidt ${ }^{11}$, R. C. W. Henderson ${ }^{17}$, E. Hennekemper ${ }^{15}$, H. Henschel ${ }^{39}$, M. Herbst ${ }^{15}$, G. Herrera ${ }^{23}$, M. Hildebrandt ${ }^{36}$, K. H. Hiller ${ }^{39}$, D. Hoffmann ${ }^{21}$, R. Horisberger ${ }^{36}$, T. Hreus ${ }^{4}$, F. Huber ${ }^{14}$, M. Jacquet ${ }^{27}$, X. Janssen ${ }^{4}$, L. Jönsson ${ }^{20}$, H. Jung ${ }^{11,4}$, M. Kapichine ${ }^{9}$, I. R. Kenyon ${ }^{3}$, C. Kiesling ${ }^{26}$, M. Klein ${ }^{18}$, C. Kleinwort ${ }^{11}$, T. Kluge ${ }^{18}$, R. Kogler $^{12}$, P. Kostka ${ }^{39}$, M. Krämer ${ }^{11}$, J. Kretzschmar ${ }^{18}$, K. Krüger ${ }^{15}$, M. P. J. Landon ${ }^{19}$, W. Lange ${ }^{39}$, G. Laštovička-Medin ${ }^{30}$, P. Laycock ${ }^{18}$, A. Lebedev ${ }^{25}$, V. Lendermann ${ }^{15}$, S. Levonian ${ }^{11}$, K. Lipka ${ }^{11}$, , B. List ${ }^{11}$, J. List ${ }^{11}$, B. Lobodzinski ${ }^{11}$, R. Lopez-Fernandez ${ }^{23}$, V. Lubimov ${ }^{24}$, E. Malinovski ${ }^{25}$, H.-U. Martyn' ${ }^{1}$, S. J. Maxfield ${ }^{18}$, A. Mehta ${ }^{18}$, A. B. Meyer ${ }^{11}$, H. Meyer $^{37}$, J. Meyer ${ }^{11}$, S. Mikocki ${ }^{7}$, I. Milcewicz-Mika ${ }^{7}$, F. Moreau ${ }^{28}$, A. Morozov', J. V. Morris ${ }^{6}$, K. Müller ${ }^{41}$, Th. Naumann ${ }^{39}$, P. R. Newman ${ }^{3}$, C. Niebuhr ${ }^{11}$, D. Nikitin ${ }^{9}$, G. Nowak ${ }^{7}$, K. Nowak ${ }^{12}$, J. E. Olsson ${ }^{11}$, D. Ozerov ${ }^{11}$, P. Pahl ${ }^{11}$, V. Palichik ${ }^{9}$, I. Panagoulias ${ }^{11, b, y}$, M. Pandurovic ${ }^{2}$, Th. Papadopoulou ${ }^{11, b, y}$, C. Pascaud ${ }^{27}$, G. D. Patel ${ }^{18}$, E. Perez ${ }^{10, e}$, A. Petrukhin $^{11}$, I. Picuric $^{30}$, H. Pirumov ${ }^{14}$, D. Pitzl ${ }^{11}$, R. Plačakytè ${ }^{11}$, B. Pokorny ${ }^{32}$, R. Polifka ${ }^{32,1}$, B. Povh ${ }^{13}$, V. Radescu ${ }^{11}$, N. Raicevic ${ }^{30}$, T. Ravdandorj $^{35}$, P. Reimer ${ }^{31}$, E. Rizvi ${ }^{19}$, P. Robmann ${ }^{41}$, R. Roosen ${ }^{4}$, A. Rostovtsev ${ }^{24}$, M. Rotaru ${ }^{5}$, J. E. Ruiz Tabasco ${ }^{22}$, S. Rusakov ${ }^{25}$, D. Sálek ${ }^{32}$, D. P. C. Sankey ${ }^{6}$, M. Sauter ${ }^{14}$, E. Sauvan'1,m, S. Schmitt11, L. Schoeffel ${ }^{10}$, A. Schöning ${ }^{14}$, H.-C. Schultz-Coulon ${ }^{15}$, F. Sefkow ${ }^{11}$, L. N. Shtarkov ${ }^{25}$, S. Shushkevich ${ }^{11}$, T. Sloan ${ }^{17}$, Y. Soloviev ${ }^{11,25}$, P. Sopicki ${ }^{7}$,

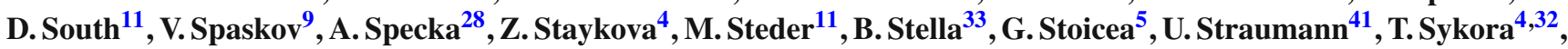
P. D. Thompson ${ }^{3}$, T. H. Tran $^{27}$, D. Traynor ${ }^{19}$, P. Truöl ${ }^{41}$, I. Tsakov ${ }^{34}$, B. Tseepeldorj ${ }^{35, i}$, J. Turnau ${ }^{7}$, A. Valkárová ${ }^{32}$, C. Vallée ${ }^{21}$, P. Van Mechelen ${ }^{4}$, Y. Vazdik ${ }^{25}$, D. Wegener ${ }^{8}$, E. Wünsch ${ }^{11}$, J. Žáček ${ }^{32}$, J. Zálešák ${ }^{31}$, Z. Zhang²7, A. Zhokin ${ }^{24}$, R. Žlebčík ${ }^{32}$, H. Zohrabyan ${ }^{38}$, F. Zomer ${ }^{27}$

\footnotetext{
${ }^{1}$ I. Physikalisches Institut der RWTH, Aachen, Germany

2 Vinca Institute of Nuclear Sciences, University of Belgrade, 1100 Belgrade, Serbia

${ }^{3}$ School of Physics and Astronomy, University of Birmingham, Birmingham, UK ${ }^{\mathrm{O}}$

${ }^{4}$ Inter-University Institute for High Energies ULB-VUB, Brussels and Universiteit Antwerpen, Antwerpen, Belgium ${ }^{p}$

${ }^{5}$ National Institute for Physics and Nuclear Engineering (NIPNE), Bucharest, Romania ${ }^{z}$

${ }^{6}$ STFC, Rutherford Appleton Laboratory, Didcot, Oxfordshire, UK ${ }^{\circ}$

${ }^{7}$ Institute for Nuclear Physics, Cracow, Poland ${ }^{q}$

${ }^{8}$ Institut für Physik, TU Dortmund, Dortmund, Germany ${ }^{\text {n }}$

${ }^{9}$ Joint Institute for Nuclear Research, Dubna, Russia

${ }^{10}$ CEA, DSM/Irfu, CE-Saclay, Gif-sur-Yvette, France

${ }^{11}$ DESY, Hamburg, Germany

${ }^{12}$ Institut für Experimentalphysik, Universität Hamburg, Hamburg, Germany ${ }^{\text {n }}$

${ }^{13}$ Max-Planck-Institut für Kernphysik, Heidelberg, Germany

${ }^{14}$ Physikalisches Institut, Universität Heidelberg, Heidelberg, Germany ${ }^{\text {n }}$

${ }^{15}$ Kirchhoff-Institut für Physik, Universität Heidelberg, Heidelberg, Germany ${ }^{\mathrm{n}}$

${ }^{16}$ Institute of Experimental Physics, Slovak Academy of Sciences, Košice, Slovak Republic ${ }^{\mathrm{s}}$

${ }^{17}$ Department of Physics, University of Lancaster, Lancaster, UK ${ }^{\circ}$

18 Department of Physics, University of Liverpool, Liverpool, $\mathrm{UK}^{\mathrm{O}}$

${ }^{19}$ School of Physics and Astronomy, Queen Mary, University of London, London, $\mathrm{UK}^{\mathrm{O}}$

${ }^{20}$ Physics Department, University of Lund, Lund, Sweden ${ }^{t}$

${ }^{21}$ CPPM, Aix-Marseille Univ., CNRS/IN2P3, 13288 Marseille, France
} 
${ }^{22}$ Departamento de Fisica Aplicada, CINVESTAV, Mérida, Yucatán, Méxicow

${ }^{23}$ Departamento de Fisica, CINVESTAV IPN, México City, Méxicow

24 Institute for Theoretical and Experimental Physics, Moscow, Russia ${ }^{\mathrm{x}}$

${ }^{25}$ Lebedev Physical Institute, Moscow, Russia

${ }^{26}$ Max-Planck-Institut für Physik, München, Germany

${ }^{27}$ LAL, Université Paris-Sud, CNRS/IN2P3, Orsay, France

${ }^{28}$ LLR, Ecole Polytechnique, CNRS/IN2P3, Palaiseau, France

${ }^{29}$ LPNHE, Université Pierre et Marie Curie Paris 6, Université Denis Diderot Paris 7, CNRS/IN2P3, Paris, France

${ }^{30}$ Faculty of Science, University of Montenegro, Podgorica, Montenegro aa

${ }^{31}$ Institute of Physics, Academy of Sciences of the Czech Republic, Praha, Czech Republic ${ }^{u}$

${ }^{32}$ Faculty of Mathematics and Physics, Charles University, Praha, Czech Republic ${ }^{\mathrm{u}}$

33 Dipartimento di Fisica, Università di Roma Tre and INFN Roma 3, Roma, Italy

${ }^{34}$ Institute for Nuclear Research and Nuclear Energy, Sofia, Bulgaria ${ }^{\mathrm{r}}$

35 Institute of Physics and Technology of the Mongolian Academy of Sciences, Ulaanbaatar, Mongolia

${ }^{36}$ Paul Scherrer Institut, Villigen, Switzerland

${ }^{37}$ Fachbereich C, Universität Wuppertal, Wuppertal, Germany

38 Yerevan Physics Institute, Yerevan, Armenia

${ }^{39}$ DESY, Zeuthen, Germany

${ }^{40}$ Institut für Teilchenphysik, ETH, Zürich, Switzerland ${ }^{\mathrm{V}}$

${ }^{41}$ Physik-Institut der Universität Zürich, Zürich, Switzerland ${ }^{v}$

Received: 26 November 2013 / Accepted: 9 December 2013 / Published online: 14 February 2014

(C) The Author(s) 2014. This article is published with open access at Springerlink.com

\section{Erratum to: Eur Phys J C (2012) 72:2163 DOI 10.1140/epjc/s10052-012-2163-2}

The calculation of the QED Compton (QEDC) cross sections in a recent $\mathrm{H} 1$ publication [1] is based on the COMPTON22 event generator $[2,3]$. The cross sections for elastic, quasielastic and inelastic QEDC are all proportional to $(\hbar c)^{2} \alpha^{3} / s$, where $\alpha$ is the fine-structure constant, $s$ is the centre-of-mass energy squared measured in $\mathrm{GeV}^{2}$ and the factor $(\hbar c)^{2}$ is a conversion factor, in units of $\left[\mathrm{pb} \mathrm{GeV}^{2}\right]$. It was found that

The online version of the original article can be found under doi:10.1140/epjc/s10052-012-2163-2.

a e-mail: daum@mail.desy.de

b Also at Physics Department, National Technical University, Zografou Campus, 15773 Athens, Greece.

c Also at Rechenzentrum, Universität Wuppertal, Wuppertal, Germany.

d Also at IPNL, Université Claude Bernard Lyon 1, CNRS/IN2P3, Villeurbanne, France.

e Also at CERN, Geneva, Switzerland.

${ }^{f}$ Also at Max-Planck-Institut für Physik, München, Germany.

g Also at Comenius University, Bratislava, Slovak Republic.

h Also at Faculty of Physics, University of Bucharest, Bucharest, Romania.

${ }^{i}$ Also at Ulaanbaatar University, Ulaanbaatar, Mongolia.

j Supported by the Initiative and Networking Fund of the Helmholtz Association (HGF) under the contract VH-NG-401.

k Absent on leave from NIPNE-HH, Bucharest, Romania.

${ }^{1}$ Also at Department of Physics, University of Toronto, Toronto, Ontario, Canada M5S 1A7.

m Also at LAPP, Université de Savoie, CNRS/IN2P3, Annecy-le-Vieux, France.

n Supported by the Bundesministerium für Bildung und Forschung, FRG, under contract numbers 05H09GUF, 05H09VHC, 05H09VHF, 05H16PEA. incorrect numerical values have been programmed in the FORTRAN code used to calculate the cross sections, namely $(\hbar c)^{2}=(6.20087)^{2} \times 10^{7} \mathrm{pb} \mathrm{GeV}^{2}$ and $\alpha=1 / 137$. These numbers are corrected to $(\hbar c)^{2}=0.389379 \times 10^{9} \mathrm{pb} \mathrm{GeV}^{2}$ and $\alpha=0.00729735$, thus enhancing the predicted QEDC cross sections by $1.19 \%$.

Furthermore, the running of the electromagnetic coupling as a function of the virtuality $t$ of the exchanged photon is neglected in [2,3]. For the reanalysis of [1], a running finestructure constant $\alpha(t)$ is implemented. The cross sections predicted by COMPTON22 are scaled by a factor $(\alpha(t) / \alpha)^{2}$ prior to integrating over $t$. The running coupling $\alpha(t)$ is evalu-

${ }^{\circ}$ Supported by the UK Science and Technology Facilities Council, and formerly by the UK Particle Physics and Astronomy Research Council.

p Supported by FNRS-FWO-Vlaanderen, IISN-IIKW and IWT and by Interuniversity Attraction Poles Programme, Belgian Science Policy.

q Partially Supported by Polish Ministry of Science and Higher Education, grant DPN/N168/DESY/2009.

${ }^{\mathrm{r}}$ Supported by the Deutsche Forschungsgemeinschaft.

s Supported by VEGA SR grant no. 2/7062/ 27.

${ }^{t}$ Supported by the Swedish Natural Science Research Council.

" Supported by the Ministry of Education of the Czech Republic under the projects LC527, INGO-LA09042 and MSM0021620859.

${ }^{v}$ Supported by the Swiss National Science Foundation.

${ }^{w}$ Supported by CONACYT, México, grant 48778-F.

${ }^{x}$ Russian Foundation for Basic Research (RFBR), grant no 1329.2008.2 and Rosatom.

y This project is co-funded by the European Social Fund (75 \%) and National Resources (25\%)-(EPEAEK II)-PYTHAGORAS II.

${ }^{\mathrm{z}}$ Supported by the Romanian National Authority for Scientific Research under the contract PN 09370101.

${ }^{\text {aa }}$ Partially Supported by Ministry of Science of Montenegro, no. 051/3-3352. 
Table 1 Updated table of background fractions obtained from the reanalysis of [1]

\begin{tabular}{llllll}
\hline & \multicolumn{2}{l}{ No $\left|\vec{P}_{T}^{\text {miss }}\right|$ cut } & & $\left|\vec{P}_{T}^{\text {miss }}\right|<0.3 \mathrm{GeV}$ & \\
\cline { 2 - 3 } & In Ref. [1] (\%) & This analysis (\%) & & In Ref. [1] (\%) & This analysis (\%) \\
\hline Quasi-elastic QEDC & 6.84 & 6.93 & 2.93 & 2.96 \\
Inelastic QEDC & 7.02 & 7.15 & 1.51 & 1.52 \\
Elastic DVCS & 2.10 & 2.06 & 1.26 & 1.24 \\
Quasi-elastic DVCS & 0.55 & 0.54 & 0.16 & 0.15 \\
$e p \rightarrow e p e^{+} e^{-}$ & 1.15 & 1.12 & 1.31 & 1.28 \\
Diffractive DIS & 2.78 & 2.72 & 0.53 & 0.52 \\
Non-diffractive DIS & 0.02 & 0.02 & 0.01 & 0.01 \\
Diffractive $\rho^{0}$ & 2.05 & 2.00 & 0.15 & 0.15 \\
Diffractive $\omega$ & 0.43 & 0.42 & 0.03 & 0.03 \\
Diffractive $\phi$ & 0.29 & 0.28 & 0.02 & 0.02 \\
Diffractive $J / \psi$ & 0.20 & 0.20 & 0.05 & 0.05 \\
Diffractive $\psi^{\prime}$ & 0.17 & 0.17 & 0.08 & 0.08 \\
Diffractive $\Upsilon$ & 0.02 & 0.02 & 0.01 & 0.01 \\
\hline
\end{tabular}

ated using the alphaQED code $[4,5]$. This change increases the predicted cross sections in the analysis phase space [1] by $(0.83 \pm 0.04) \%$, where the uncertainty is related to the number of generated events.

Taking both effects together, the predicted elastic QEDC cross section in the analysis phase space is increased by $2.0 \%$. The corrected cross sections in the generated phase space is $\sigma_{\text {gen }}=55.9 \mathrm{pb}$, where the estimated uncertainty on the QEDC theory of $1.1 \%$ is unchanged. Similarly, the visible cross section is increased to $\sigma_{\mathrm{vis}}=37.1 \mathrm{pb}$. The background fractions also change slightly, as shown in Table 1. Background from quasi-elastic and inelastic QEDC processes increases, because the $t$ dependence is different from that of the elastic QEDC process. The relative fractions of the other background sources are reduced, because their predicted absolute cross sections do not change.

In addition to the above changes in the cross section prediction, a small inefficiency in the data handling has been identified. After correcting this technical problem, 21 additional data events are recovered for the luminosity measurement, now derived from a total of 14,298 candidate events.

The overall HERA luminosity in the data taking period from 2003 to 2007, measured from counting QED Compton events, is found to be $345.3 \pm 7.9 \mathrm{pb}^{-1}$. As compared to [1] it is lower by $1.8 \%$. The cross section measurements performed in three $\mathrm{H} 1$ papers [6-8], based on the data collected in the years 2003-2007 at a proton energy of $920 \mathrm{GeV}$, are normalised using the integrated luminosity measurement of [1]. For this reason they are affected by the change in the measured luminosity discussed above, such that their cross sections are to be scaled up by $1.8 \%$. It is worth to note that these changes are fully covered by the total uncertainty of the luminosity measurement of $2.3 \%$. The measurements of beauty production at threshold [6] and of elastic and proton- dissociative $J / \psi$ production [8] have systematic uncertainties which are much larger than the correction discussed above and are not updated. In contrast, the measurements of inclusive neutral and charged current cross sections [7] reach a level of precision where the $1.8 \%$ correction may be of relevance. Furthermore, the combined data tables 29-32, 45-48 and 51-52 in [7], cannot be derived using a simple scale factor, because other datasets, not affected by the problems discussed above, are included in the averaging procedure. The corrected data tables of [7] are available [9].

Acknowledgements We would like to thank Prof. H. Spiesberger for useful discussions on the running of the fine-structure constant.

Open Access This article is distributed under the terms of the Creative Commons Attribution License which permits any use, distribution, and reproduction in any medium, provided the original author(s) and the source are credited.

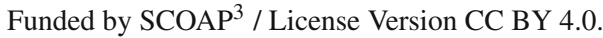

\section{References}

1. F.D. Aaron et al. [H1 Collaboration], Eur. Phys. J. C 72, 2163 (2012), [arXiv:1205.2448]

2. A. Courau, P. Kessler, Phys. Rev. D 46, 117 (1992)

3. V. Lendermann, H.C. Schultz-Coulon, D. Wegener, Eur. Phys. J. C 31, 343 (2003). [arXiv:hep-ph/0307116]

4. F. Jegerlehner, Nuovo Cim. C 034S1, 31 (2011). [arXiv:1107.4683]

5. alphaQED, Version of April 5, 2012. http://www-com.physik. hu-berlin.de/ fjeger/software.html

6. F.D. Aaron et al. [H1 Collaboration], Eur. Phys. J. C 72, 2148 (2012). [arXiv:1206.4346]

7. F.D. Aaron et al. [H1 Collaboration], JHEP 1209, 061 (2012). [arXiv: 1206.7007]

8. C. Alexa et al. [H1 Collaboration], Eur. Phys. J. C 73, 2466 (2013). [arXiv:1304.5162]

9. H1 collaboration, list of publications. https://www-h1.desy.de/ publications/H1publication.short_list.html 